\title{
CROSTAS BIOLÓGICAS DE SAPRÓLITOS DA REGIÃO DO QUADRILATTERO FERRÍFERO, MG: CICLAGEM BIOGEOQUÍMICA E MICROMORFOLÓGICA
}

\author{
Elaine de S. Trindade (*), Carlos Ernesto G.R. Schaefer (**), Walter A. P. Abrahão (***), \\ Emerson S. Ribeiro Jr (****), Deise M.F. Oliveira (****) \& Paulo César Teixeira (****)
}

\begin{abstract}
The role of biological crusts in nutrient cycling and soil structure genesis in saprolites, in tropical conditions, is little known. This work reports on the biochemical weathering and structural reorganization resulting from the biological crusts action in different saprolites of the Quadrilátero Ferrifero and neighbor areas, MG. These road exposures were oriented to the south, encompassing a diabase (DB), gneiss (GN), itabirite (IT), schist (XT) and gold-mine spoil-pile from Morro Velho (MV), comprising metasediments of the Rio das Velhas supergroup. There were collected and identified cyanobacteria, fungi, lichens and mosses. Based on the biogeochemical study, the exchangeable levels of $\mathrm{K}, \mathrm{Mn}$ and $\mathrm{Zn}$ were preferably concentrated in the crust. The exchangeable Fe levels were higher in the altered under zone, constituted possibly by secondary Fe-migration and precipitation through lateral fluxes. There were no clear trend of $\mathrm{Ca}$ and $\mathrm{Mg}$ distribution, although they were apparently concentrated in the crust in the IT and XT substrates, and in the altered zone in the DB. In MV spoil, the Ca dynamic was affected by sulfide oxidation, following acid drainage. In terms of total levels Mn, $\mathrm{Fe}, \mathrm{Mg}, \mathrm{Cu}$ and $\mathrm{Zn}$ were higher in the diabase saprolite, due to its maficity, in a clear contrast with the host gneiss, represented by the GN saprolite, with low levels of $\mathrm{Fe}, \mathrm{Mn}, \mathrm{Mg}$ and $\mathrm{Cu}$. The richer substrates (DB, MV e XT) showed a trend of increasing total levels with depth, whilst the nutrient poor substrates (GN e IT) had higher levels of some elements in the crust, compared with the saprolito. The micropedological features are notably rich and varied, showed buried micropedogenetic horizons, sapric and histic micro-horizons, micro-stratification, ferruginous zones similar to $\mathrm{Fe} / \mathrm{Mn}$ micropans, amongst others. The biological crusts efficiently covered the saprolites, being responsible to micropedogenetic features and a clear process of biological microstructuration of the exposed saprolites. This process is capable of altering the moisture balance and erosion dynamic of the slope, with important geomorphological and environmental implications.
\end{abstract}

\section{INTRODUÇÃO}

Grande parte dos estudos voltados para o entendimento das interações entre microrganismos e substrato inorgânico tem sido conduzida por biólogos e geólogos, sobretudo no que se refere à atividade de líquens. Consequentemente, pouco do significado da ciclagem biogeoquímica na gênese do solo sob crostas biológicas foi até agora esclarecido.

Por crosta biológica entende-se uma fina camada orgânica, com espessura entre 5 e 50mm (Pérez, 1997), formada à superfície de substratos de diversas naturezas (rocha inalterada, saprolito ou solo) em função da instalação e desenvolvimento de comunidades de microrganismos e plantas inferiores, que podem compreender bactérias, fungos, algas, líquens, briófitas e, ou, pteridófitas.

Trabalhos específicos sobre o tema apontam para o papel da biota no intemperismo biofísico do substrato mineral, principalmente através da penetração de hifas e filamentos e consequente desagregação associada aos ciclos de hidratação- desidratação (Jones et al., 1987, Wierzchos e Ascaso, 1998; Barker et al., 1998). Por outro lado, há trabalhos que ressaltam a importância dessas comunidades pioneiras na estabilização de solos sobre os quais são esparsas ou ausentes plantas superiores (Neuman et al., 1996; Pérez, 1997; Barker et al., 1998). Nesse sentido, são levantadas como principais causas do aumento da resistência à erosão, em relação aos sítios descobertos, o aumento da infiltração e manutenção de umidade, condicionados pela rugosidade e concentração de compostos orgânicos na superfície do encrostamento, e maior coesão das partículas do solo, proporcionada por cimentantes orgânicos (principalmente, polissacarídeos) e crescimento de corpos filamentosos. Inúmeros trabalhos confirmam a ênfase dada ao papel das associações organo-minerais na agregação e estabilidade estrutural dos solos, embora não sejam específicos sobre encrostamentos orgânicos (Martin e Waksman, 1940; Peele e Beale, 1941; Tisdall e Oades, 1982; Lynch e Elliott, 1983; Chenu, 1989 e 1993; Dorioz et al. 1993; Emerson, 1995; Faria,1996).

$\left(^{*}\right)$ Geógrafa, mestre em Solos e Nutrição de Plantas pelo Depto de Solos $\left(^{* *}\right)$ Professor Adjunto do Depto de Solos $\left.{ }^{(* * *}\right)$ Professor Assistente do Depto de Solos $\left(^{* * * *}\right.$ Engenheiros agrônomos, doutorandos do Depto de Solos. Universidade Federal de Viçosa, Viçosa, 36.571-000, MG, Brasil. E-mail: carlos.schaefer@solos.ufv.br. 
Além do relevante papel na estabilização da incipiente estrutura do solo formado, as algas cianofíceas ou cianobactérias são importantes colonizadores fotoautotróficos da superfície externa, sintetizando compostos orgânicos, que podem ser utilizados por outros organismos, e fixando nitrogênio atmosférico (Zimmerman, 1993).

Os efeitos da atividade biológica no processo de intemperismo bioquímico têm sido atribuídos à excreção de ácidos orgânicos e formação de quelatos com componentes inorgânicos do substrato imediato, propiciando a decomposição e neosíntese de minerais de argila na interface substrato-crosta (Weed et al., 1969; Iskandar e Syers, 1972; Brown, 1976; Williams e Robinson, 1994; Viles e Pentecost, 1994; Barker et al., 1998; Wierzchos e Ascaso, 1998; Ehrlich, 1998). Em geral, informações disponíveis na literatura sobre ciclagem biogeoquímica associada a microrganismos referem-se, entretanto, a organismos específicos, atuando sobre substratos também específicos. Dados encontrados sobre o comportamento de líquens normalmente são de interesse para o campo da microbiologia. Em relação a bactérias e fungos, atenção maior tem sido dirigida para seu papel na decomposição de resíduos orgânicos e, consequentemente, na ciclagem de carbono e nitrogênio, em áreas submetidas a diferentes sistemas de preparo do solo.

No presente trabalho, estudou-se a ciclagem biogeoquímica e a microestruturação biológica em microambientes associados à presença de crostas biológicas, com o objetivo de identificar sua relevância na gênese incipiente do solo em cortes de saprolitos expostos em taludes de estradas situadas na região do Quadrilátero Ferrífero, Minas Gerais, e adjacências. $\mathrm{Na}$ escolha das áreas de amostragem considerou-se a diversidade litológica existente em curto trajeto, o que atende as exigências de um trabalho preliminar, de caráter mais abrangente. Foram avaliados teores de macro e micronutrientes de cinco diferentes sítios cobertos por crostas, considerando-se, a priori, que substratos diferentes quanto à natureza química determinam interações contrastantes entre componentes biológicos e substratos inorgânicos.

\section{MATERIALE MÉTODOS}

Os sítios estudados no presente trabalho situam-se na região do Quadrilátero Ferrífero, Minas Gerais, e adjacências, estando compreendidos entre as coordenadas $19^{\circ} 58^{\prime} 51^{\prime \prime} / 20^{\circ} 45^{\prime} 02^{\prime \prime} \mathrm{S}$ e $42^{\circ} 52^{\prime} 12^{\prime \prime} /$ $43^{\circ} 52^{\prime} 01^{\prime \prime}$ WGr (Figura 1).

Os diferentes substratos referem-se a saprolitos desenvolvidos de itabirito (IT); xisto (XT); diabásio (DB); gnaisse (GN); e depósito de rejeito de mineração de ouro da mina de Morro Velho (MV), rico em sulfato de cálcio, correspondendo a metas-sedimentos do Supergrupo Rio das Velhas (Ladeira, 1980).

Apenas os taludes de exposição sul foram amostrados, tendo sido observado um desenvolvimento mais expressivo das crostas nesta orientação. As coletas foram feitas através de microtrincheiras, sendo discriminadas amostras da cobertura orgânica propriamente dita, camada intermediária (aproximadamente $0-5 \mathrm{~mm}$ de profundidade) e saprolito (abaixo de $50 \mathrm{~mm}$ ). As briófitas foram os organismos dominantes na composição das crostas (Quadro 1), exceto sobre o substrato gnáissico, sendo a Cladonia $s p$. a única espécie de líquen identificada.

Análises químicas de rotina referentes a $\mathrm{pH}$ em água (relação 1:2,5), determinação dos teores disponíveis de $\mathrm{P}, \mathrm{K}, \mathrm{Zn}, \mathrm{Fe}, \mathrm{Mn}$ e $\mathrm{Cu}$ (extrator Mehlich 1) e dos teores trocáveis de $\mathrm{Ca}$ e $\mathrm{Mg}$ (extrator $\mathrm{KCl} 1 \mathrm{~mol} / \mathrm{L}$ ) foram realizadas em amostras da camada intermediária e saprolito. Amostras relativas à cobertura orgânica foram submetidas à digestão nitroperclórica e amostras da camada intermediária e saprolito, ao ataque triácido $\left(\mathrm{HNO}_{3}, \mathrm{HClO}_{4} \mathrm{e} \mathrm{HF}\right)$, conforme EMBRAPA (1997), para a determinação de elementos totais $(\mathrm{P}, \mathrm{K}, \mathrm{Ca}, \mathrm{Mg}, \mathrm{Zn}$, $\mathrm{Fe}, \mathrm{Mn}$ e $\mathrm{Cu}$ ) e estimativa do efeito biótico sobre a reserva mineral do substrato. $\mathrm{O}$ teor total de $\mathrm{C}$ orgânico na crosta biológica foi obtido pela queima das amostras em mufla, a $400^{\circ} \mathrm{C}$ por quatro horas.

Para a avaliação das feições micromorfológicas e pedogenéticas da crosta biológica e do saprolito subjacente, amostras indeformadas foram amostradas e impregnadas com resina de poliéster REVOPAL T208, contendo corante fluorescente (Uvitex OB;Ciba-Geigy). Foram confeccionadas lâminas delgadas no sentido transversal à crosta microbiótica, as quais foram observadas em microscópio petrográfico (modelo OLYMPUS DX40) e suas feições micropedológicas descritas segundo FitzPatrick (1993). As fotografias foram feitas em filme Kodacolor, 35mm, ISO 100.

\section{RESULTADOS E DISCUSSÃO}

\section{Ciclagem biogeoquímica}

Saprolito: teores disponiveis

As análises químicas dos saprolitos evidenciaram valores gerais mais elevados de nutrientes disponíveis no microperfil da Mina de Morro Velho (MV), notadamente de $\mathrm{P}, \mathrm{Ca}, \mathrm{Fe}, \mathrm{Zn}$ e $\mathrm{Cu}$, com valores relativamente menores de $\mathrm{K}$ e $\mathrm{Mn}$ (Quadro 2). Provavelmente estes valores tenham sido influenciados pela formação de precipitados em ambiente submetido à oxidação de sulfetos.

Para K, os teores trocáveis foram máximos no saprolito de gnaisse (GN), possivelmente pela abundância observada de biotita/ilita. No xisto sericítico (XT), os teores de $\mathrm{K}$ disponível foram surpreendentemente baixos, indicando, provavelmente, o extremo grau de alteração sofrido por este material. Os menores valores de $\mathrm{K}$ disponível foram encontrados no itabirito (IT), o que deve estar sendo condicionado pela pobreza do saprolito em minerais potássicos. Os teores de Mg trocáveis foram baixos, exceto no saprolito de diabásio (DB), pela natureza máfica do substrato (Gomes, 1988). 


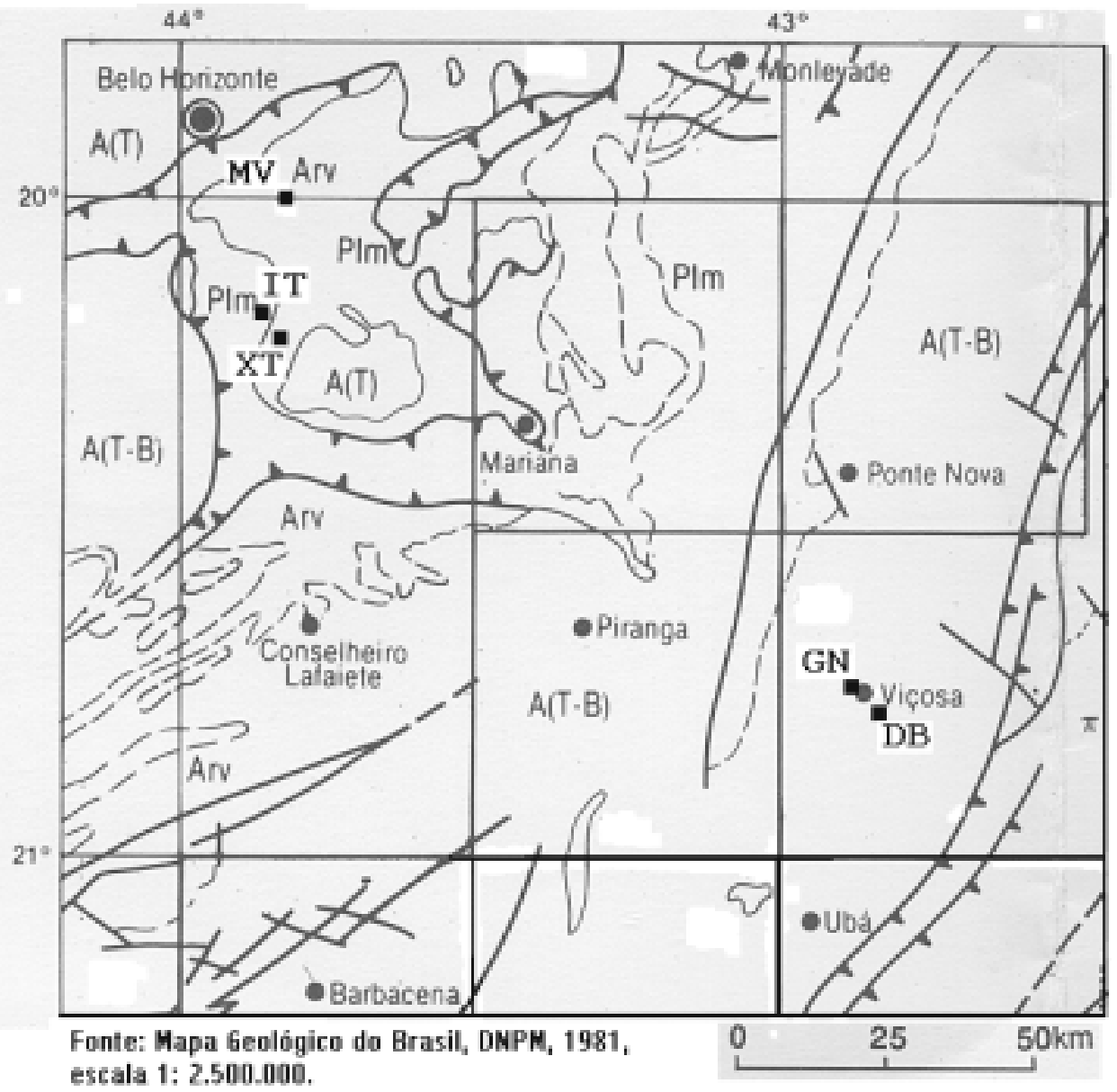
A (T) - Arqueano indiferenciado retrabalhado no Ciclo Transamazônico
A (T-B) - Arqueano indiferenciado retrabalhado nos Ciclos Transamazônico
e Brasiliano; (T) charnoquito
Arv - Supergrupo Rio das Velhas
PIm - Supergrupo Minas

Figura 1 : Locais selecionados para amostragem.

Figure 1: Selected places to sampling.

\begin{tabular}{|c|c|c|c|c|c|}
\hline Substrato & $\begin{array}{l}\text { Altitude } \\
\text { (m) }\end{array}$ & $\begin{array}{l}\text { Direção } \\
\text { talude }\end{array}$ & $\begin{array}{l}\text { Eixo } \\
\text { talude }\end{array}$ & $\begin{array}{l}\text { Inclinação } \\
\text { talude }\end{array}$ & Composição da crosta ${ }^{1}$ \\
\hline MV & 728 & SW & N70W & $35^{\circ}$ & $\begin{array}{l}\text { Dicranella hilariana (Mont.) Mitt. } \\
\text { Cladonia sp. }\end{array}$ \\
\hline IT & 1250 & SE & $\mathrm{N} 80 \mathrm{E}$ & $80^{\circ}$ & $\begin{array}{l}\text { Polytrichum juniperinum Willd. Ex } \\
\text { Hedw. } \\
\text { Dicranella hilariana (Mont.) Mitt. } \\
\text { Bryum } s p . \\
\text { Cladonia } s p \text {. }\end{array}$ \\
\hline $\mathrm{XT}$ & 1080 & SW & $\mathrm{N} 60 \mathrm{~W}$ & $60^{\circ}$ & $\begin{array}{l}\text { Campylopus jovén } \\
\text { Filamentos de fungo não identificado e } \\
\text { algas cianobactérias }\end{array}$ \\
\hline DB & 600 & SE & $\mathrm{N} 35 \mathrm{E}$ & $55^{\circ}$ & Funaria hygrometrica Hedw. \\
\hline GN & 660 & SE & N70E & $85^{\circ}$ & $\begin{array}{l}\text { Líquen pulverulento não identificado } \\
\text { Massa de alga filamentosa (cianobactéria) }\end{array}$ \\
\hline
\end{tabular}

1 Espécies identificadas pela Dra. Olga Yano do Instituto de Botânica de São Paulo, SP, em comunicação pessoal.

Quadro 1: Caracterização dos taludes e composição das crostas biológicas.

Chart1: Characterization of cuts and composition of biological crusts. 


\begin{tabular}{|c|c|c|c|c|c|c|c|c|c|}
\hline \multirow[t]{2}{*}{ Substrato } & pH & $\mathbf{P}$ & $\mathbf{K}$ & $\mathbf{C a}$ & Mg & $\mathbf{F e}$ & Mn & $\mathbf{Z n}$ & $\mathrm{Cu}$ \\
\hline & $\mathrm{H}_{2} \mathrm{O}$ & \multicolumn{2}{|c|}{$\mathrm{mg} / \mathrm{kg}$} & \multicolumn{2}{|c|}{$\mathrm{cmol}_{\mathrm{c}} / \mathrm{kg}$} & \multicolumn{4}{|c|}{$\mathrm{mg} / \mathrm{kg}$} \\
\hline \multicolumn{10}{|l|}{ MorroVelho } \\
\hline Camada org. & & 4,57 & 71,73 & 0,00 & 2,23 & 0,00 & 176,12 & 0,21 & 9,99 \\
\hline 0 a $5 \mathrm{~mm}$ & $3 \overline{3,85}$ & 26,38 & 33,30 & 2,79 & 0,68 & 185,56 & 36,10 & 1,43 & 12,36 \\
\hline$>50 \mathrm{~mm}$ & 3,10 & 31,28 & 8,83 & 23,24 & 0,83 & 211,69 & 4,29 & 1,93 & 6,97 \\
\hline \multicolumn{10}{|l|}{ Itabirito } \\
\hline Camada org. & & 2,93 & 40,44 & 2,63 & 0,51 & 42,54 & 771,39 & 7,55 & 0,21 \\
\hline 0 a $5 \mathrm{~mm}$ & $3 \overline{, 85}$ & 16,62 & 13,09 & 1,33 & 0,27 & 136,20 & 116,44 & 4,09 & 2,34 \\
\hline$>50 \mathrm{~mm}$ & 4,95 & 17,77 & 0,67 & 0,62 & 0,00 & 77,04 & 7,88 & 0,43 & 1,68 \\
\hline \multicolumn{10}{|l|}{ Xisto } \\
\hline Camada org. & & 1,70 & 75,69 & 0,68 & 0,35 & 9,88 & 56,47 & 11,40 & 1,48 \\
\hline 0 a $5 \mathrm{~mm}$ & 3,85 & 1,84 & 21,16 & 0,41 & 0,09 & 135,75 & 31,23 & 2,32 & 1,29 \\
\hline$>50 \mathrm{~mm}$ & 4,25 & 3,59 & 3,52 & 0,19 & 0,00 & 67,30 & 4,90 & 1,05 & 1,03 \\
\hline \multicolumn{10}{|l|}{ Diabásio } \\
\hline Camada org. & & 0,45 & 50,13 & 1,13 & 0,74 & 0,00 & 65,82 & 2,93 & 2,80 \\
\hline 0 a $5 \mathrm{~mm}$ & $3 \overline{3,85}$ & 2,83 & 77,22 & 1,44 & 2,34 & 138,92 & 35,28 & 1,99 & 3,37 \\
\hline$>50 \mathrm{~mm}$ & 4,55 & 3,49 & 19,10 & 0,25 & 1,98 & 75,53 & 4,07 & 0,83 & 3,14 \\
\hline \multicolumn{10}{|l|}{ Gnaisse } \\
\hline Camada org. & & 2,37 & 232,71 & 1,50 & 0,59 & 9,93 & 56,47 & 11,40 & 1,48 \\
\hline 0 a $5 \mathrm{~mm}$ & $3 \overline{3,85}$ & 6,35 & 151,58 & 3,71 & 1,21 & 63,25 & 74,71 & 3,85 & 1,35 \\
\hline$>50 \mathrm{~mm}$ & 4,50 & 2,31 & 36,60 & 0,92 & 0,44 & 26,00 & 3,37 & 0,24 & 1,20 \\
\hline
\end{tabular}

_ Dados não disponíveis

Quadro 2: Teores disponíveis de macro e micronutrientes, segundo as faixas de amostragem nos diferentes substratos.

Chart 2: Macro and micronutrients contents according with the sampling range into different substratum.

Quanto aos micronutrientes, Fe e Mn apresentaram teores disponíveis mais elevados no itabirito (IT), enquanto $\mathrm{Cu}$ foi maioremMV,eZnnoXTeGN.OPdisponível mostrou valores maiores em MV e IT, com níveis baixos nos demais pontos.

Crosta biológica e camada intermediária: teores disponíveis

$\mathrm{O}$ elemento mais consistentemente associado à ciclagem, tomando-se os teores disponíveis, foi o $\mathrm{K}$, que tendeu a concentrações mais elevadas na crosta (MV, IT, XT, GN), ou abaixo dela (DB) decrescendo em profundidade. O P disponível apresentou uma notável tendência à redução do saprolito para a crosta tendo em vista que a extração por Mehlich não solubiliza o $\mathrm{P}$ da biomassa microbiana nem o compartimento orgânico (P orgânico). Apenas no GN houve valores disponíveis maiores na camada abaixo da crosta. Desta forma, não é possível determinar, a partir dos dados obtidos, a extensão em que outros nutrientes estão limitando mais que o P na produção de biomassa pelas crostas biológicas. De qualquer forma, é necessário conhecer em maior detalhe as frações orgânicas e inorgânicas de $\mathrm{P}$ nas crostas e nos substratos, para uma melhor compreensão da dinâmica de $\mathrm{P}$ nas crostas.

Independente dos teores de $\mathrm{Mg}$ e das relações $\mathrm{Ca}: \mathrm{Mg}$ nos saprolitos, o $\mathrm{Ca}$ tendeu a valores sempre superiores ao Mg na crosta, em virtude da ciclagem biogeoquímica. Aúnica exceção foi a crosta de MV, devido à precipitação de $\mathrm{Ca}$ como sulfato abaixo da crosta, não apresentando valores mensuráveis de Ca trocáveis na crosta. EmMV, entretanto, os teores totais de Ca foram bem superiores aos de Mg, como se pode verificar a seguir (Quadro 3).

\section{Teores totais}

Os diferentes materiais saprolíticos mostram considerável variabilidade nos teores totais de alguns nutrientes (Quadro 3). No DB, os maiores teores totais, em relação aos demais pontos, foram de $\mathrm{Mn}, \mathrm{Cu}, \mathrm{Fe}$ e Zn, revelando o caráter máfico do material parental e a presença desses elementos ligados aos minerais secundários (argilas) ou primários resistentes. Teores totais baixos de $\mathrm{Ca}$ e $\mathrm{K}$ provavelmente se devem à intensa lixiviação desses elementos a partir do intemperismo de anfibólios (ricos em $\mathrm{Ca}$ ) e rara mica (fonte de $\mathrm{K}$ ), não havendo minerais secundários resistentes em que tais elementos possam constituir reserva. Excetuando-se o substrato de MV, os teores totais de $\mathrm{P}$ na crosta do DB foram maiores que nos demais saprolitos, em função da maficidade do substrato. Em MV, os teores elevados de P total podem estar refletindo maior solubilização de formas pouco disponíveis, devido à acidez do material.

No XT, o K apresentou os maiores teores, conforme esperado pela possível reserva de $\mathrm{K}$ na ilita presente. 


\begin{tabular}{|c|c|c|c|c|c|c|c|c|c|}
\hline Substrato & \multicolumn{5}{|c|}{ dag $/ \mathrm{kg}$} & \multicolumn{4}{|c|}{ mg/kg } \\
\hline \multicolumn{10}{|l|}{ Morro Velho } \\
\hline Camada org. & 4,24 & 0,26 & 0,11 & 0,95 & 0,32 & $141.675,00$ & 768,80 & 168,8 & 209,60 \\
\hline 0 a $5 \mathrm{~mm}$ & , & 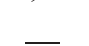 & 0,44 & 0,53 & 0,45 & $282.406,67$ & 737,80 & & 227,80 \\
\hline$>50 \mathrm{~mm}$ & - & - & 0,38 & 1,13 & 0,49 & $304.093,33$ & 805,50 & - & 218,47 \\
\hline \multicolumn{10}{|l|}{ Itabirito } \\
\hline Camada org. & 0,44 & 0,07 & 0,04 & 0,37 & 0,07 & $193.362,50$ & 898,80 & 155,00 & 43,00 \\
\hline 0 a $5 \mathrm{~mm}$ & 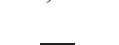 & (a) & 0,02 & 0,37 & 0,04 & $483.453,33$ & 611,83 & s. & 69,23 \\
\hline$>50 \mathrm{~mm}$ & & & 0,02 & 0,45 & 0,05 & $550.946,67$ & 611,57 & & 76,47 \\
\hline \multicolumn{10}{|l|}{ Xisto } \\
\hline Camada org. & 9,40 & 0,04 & 0,10 & 0,15 & 0,04 & $89.862,50$ & 420,00 & 91,30 & 99,10 \\
\hline 0 a $5 \mathrm{~mm}$ & s. & (n) & 1,66 & 0,44 & 0,09 & $124.246,67$ & $1.684,33$ & & 188,00 \\
\hline$>50 \mathrm{~mm}$ & - & - & 1,64 & 0,41 & 0,10 & $99.740,00$ & 579,33 & - & 176,07 \\
\hline \multicolumn{10}{|l|}{ Diabásio } \\
\hline Camada org. & 3,15 & 0.09 & 0,08 & 0,07 & 0,16 & $83.387,50$ & 260,50 & 218,80 & 198,30 \\
\hline 0 a $5 \mathrm{~mm}$ & & & 0,08 & 0,40 & 0,27 & $166.020,00$ & 619,40 & & 191,27 \\
\hline$>50 \mathrm{~mm}$ & - & - & 0,15 & 0,35 & 0,26 & $167.386,67$ & 748,77 & - & 207,97 \\
\hline \multicolumn{10}{|l|}{ Gnaisse } \\
\hline Camada org. & 2,64 & 0,06 & 0,17 & 0,46 & 0,19 & $45.150,00$ & 378,30 & 133,80 & 60,40 \\
\hline 0 a $5 \mathrm{~mm}$ & 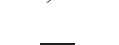 & (a) & 0,30 & 0,45 & 0,16 & $45.893,33$ & 499,70 & & 48,63 \\
\hline$>50 \mathrm{~mm}$ & & & 0,23 & 0,37 & 0,07 & $40.280,00$ & 212,50 & & 50,20 \\
\hline
\end{tabular}

_ Dados não disponíveis

Quadro 3: Teores totais de macro e micronutrientes, segundo as faixas de amostragem nos diferentes substratos.

Chart 3: Macro and micronutrients contents according with the sampling range into different substratum.

Teores totais relativamente altos de $\mathrm{Ca}$ e $\mathrm{Cu}$ foram também observados.

No IT, os maiores teores totais foram de $\mathrm{Fe}$ e $\mathrm{Ca}$, este último surpreendentemente elevado em todas as profundidades, apesar do $\mathrm{pH}$ ligeiramente menos ácido deste substrato em relação aos demais (Quadro 2). É possível que o itabirito seja localmente influenciado por fácies carbonáticas, reconhecidas no domínio do Supergrupo Minas (T. M. Dossin, comunicação pessoal), no qual se insere. Por outro lado, conforme esperado, os teores de $\mathrm{Mg}$ e $\mathrm{K}$ foram muito baixos, como reflexo da pobreza em minerais magnesianos e micas no substrato.

$\mathrm{O}$ GN mostrou valores totais baixos de Fe, $\mathrm{Mn}, \mathrm{Cu}$ e $\mathrm{Mg}$, evidenciando um notável contraste geoquímico com o DB nele intrudido.

Em termos gerais, observou-se que os substratos quimicamente mais pobres, como o gnaisse e itabirito, mostraram uma tendência a maiores teores totais na crosta (camada orgânica), em relação ao substrato mais profundo $(>50 \mathrm{~mm})$, para alguns nutrientes $(\mathrm{K}, \mathrm{Mg}$ e $\mathrm{Mn}$, no itabirito, e $\mathrm{Ca}, \mathrm{Mg}, \mathrm{Fe}, \mathrm{Mn}$ e $\mathrm{Cu}$, no gnaisse), o que reflete uma eficiente ciclagem biogeoquímica associada à crosta. Todos os demais substratos evidenciaram teores totais mais altos em profundidade. Neste caso, a fertilidade herdada do material parental sobrepuja os efeitos da ciclagem de nutrientes por influência biológica.

\section{Observações micropedológicas}

Há uma diversidade notável de morfologias entre as diferentes crostas estudadas, e os principais aspectos estão ilustrados nas Figuras 2 e 3. Em todos os casos, observou-se uma seqüência micropedogenética evidente, com diferenciação entre a camada biológica propriamente dita (crosta) na superfície, seguida de uma sub-crosta com colorações variadas e estruturação pedobiológica, e com o saprolito abaixo praticamente apédico, ou seja, com pouca estruturação.

A amostra de crosta sobre saprolito de gnaisse (GN) evidenciou um abundante microhabitat superficial de musgos/algas que fluorescem em luz polarizada, caracterizados por uma sequência descontínua de tapetes algais (algalmatts), bastante fraturados pelo umedecimento e secagem a que se encontram submetidos (Figura 2 A). Em meio ao micro-horizonte fíbrico de musgos e algas, ocorrem áreas mais frouxas e estruturadas onde se desenvolvem hifas fúngicas (seta Figura $2 \mathrm{~A}$ ), responsáveis pela degradação microbiana de resíduos ricos em lignina e celulose. De todos os saprolitos estudados, o GN mostrou-se mais rico em fungos e algalmatts que os demais, com raras briófitas.

Na crosta microbiótica sobre diabásio(DB), evidenciouse uma extensa microestruturação pela atividade microbiótica (seta superior, Figura $2 \mathrm{C}$ ), de até $12 \mathrm{~mm}$ de profundidade, com uma zona ferruginizada no contato entre a parte estruturada e o saprolito apédico (seta inferior, Figura $2 \mathrm{C}$ ). A agregação e abundante porosidade é resultante da atividade combinada de algas e musgos. Há ocorrência de micronódulos oxídicos, provavelmente de Fe e Mn, dispersos na matriz, corroborando os valores totais elevados desses elementos no saprolito (Quadro 3). 

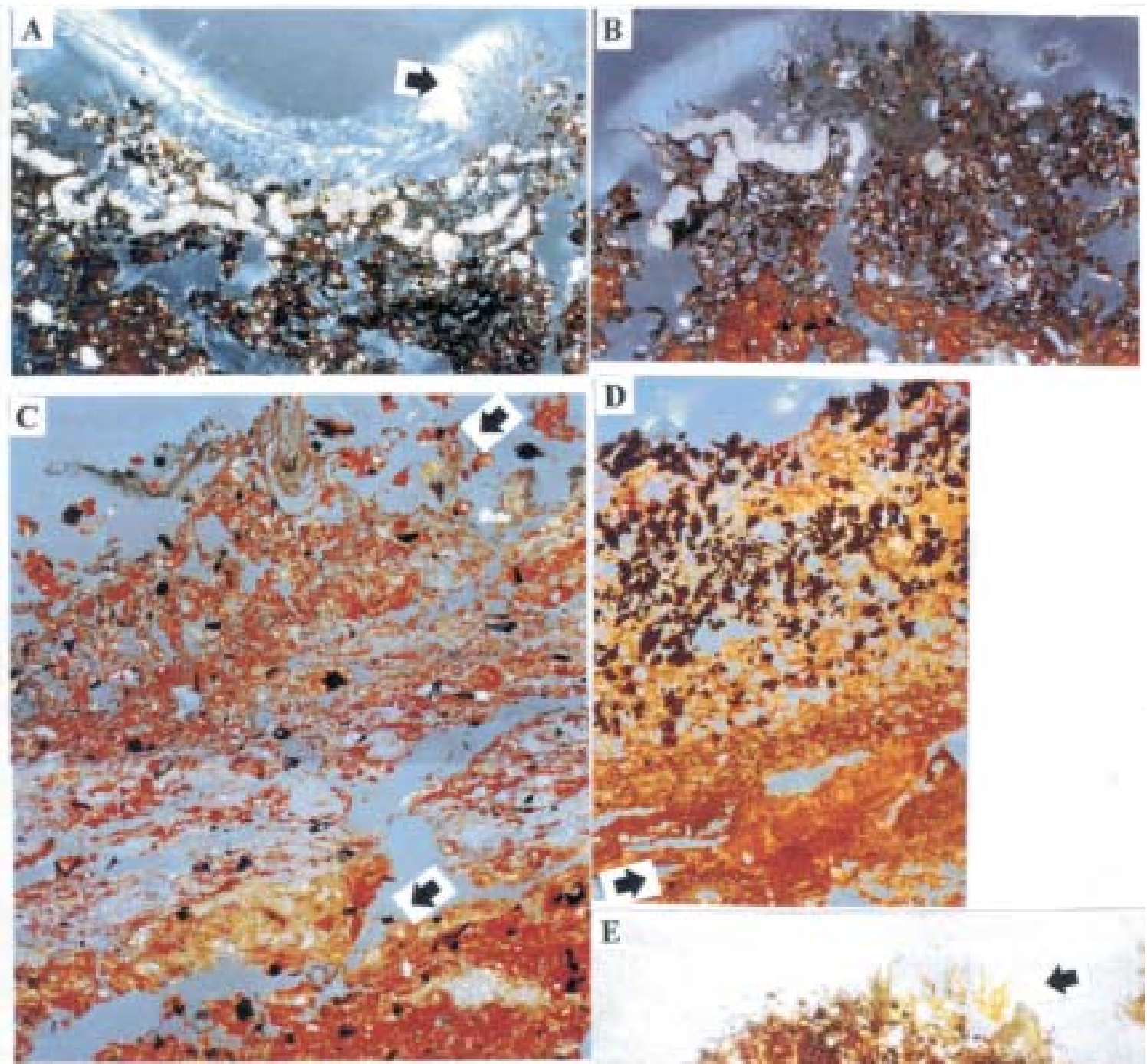

\section{E}
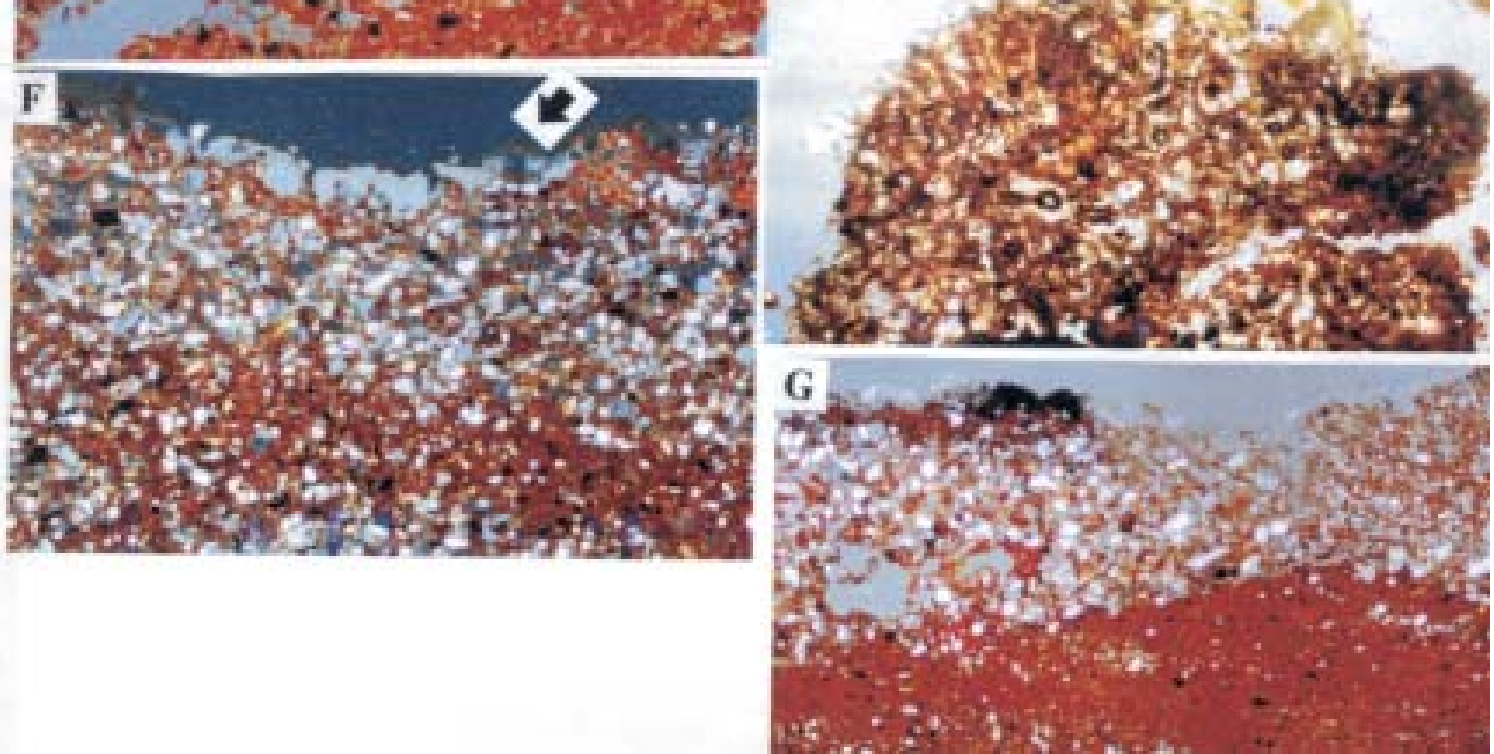

Figura 2 - A) Crosta sobre saprolito de GN, evidenciando sequência descontínua de tapetes algais (algalmatts). Hifas fúngicas são observadas em meio ao micro-horizonte fibrico (seta); B) micro-horizonte fibrico delgado e descontínuo, na amostra de $M V$; C) crosta microbiótica sobre DB, evidenciando extensa microestruturação pela atividade microbiótica (seta superior), com zona ferruginizada no contato entre a parte estruturada e o saprolito apédico (seta inferior); D) cobertura biológica rala e descontínua sobre o saprolito de IT, com áreas estruturadas onde são abundantes micropartículas oxídicas, sobrepostas à matriz fortemente ferruginizada e cimentada, abaixo (seta); E) concentração de areia fina quartzosa e delgada cobertura de algalmatts descontínua (MV); F) e G) zona maciça e pouco estruturada, com ocorrência de crosta biológica de penetração limitada (MV). 

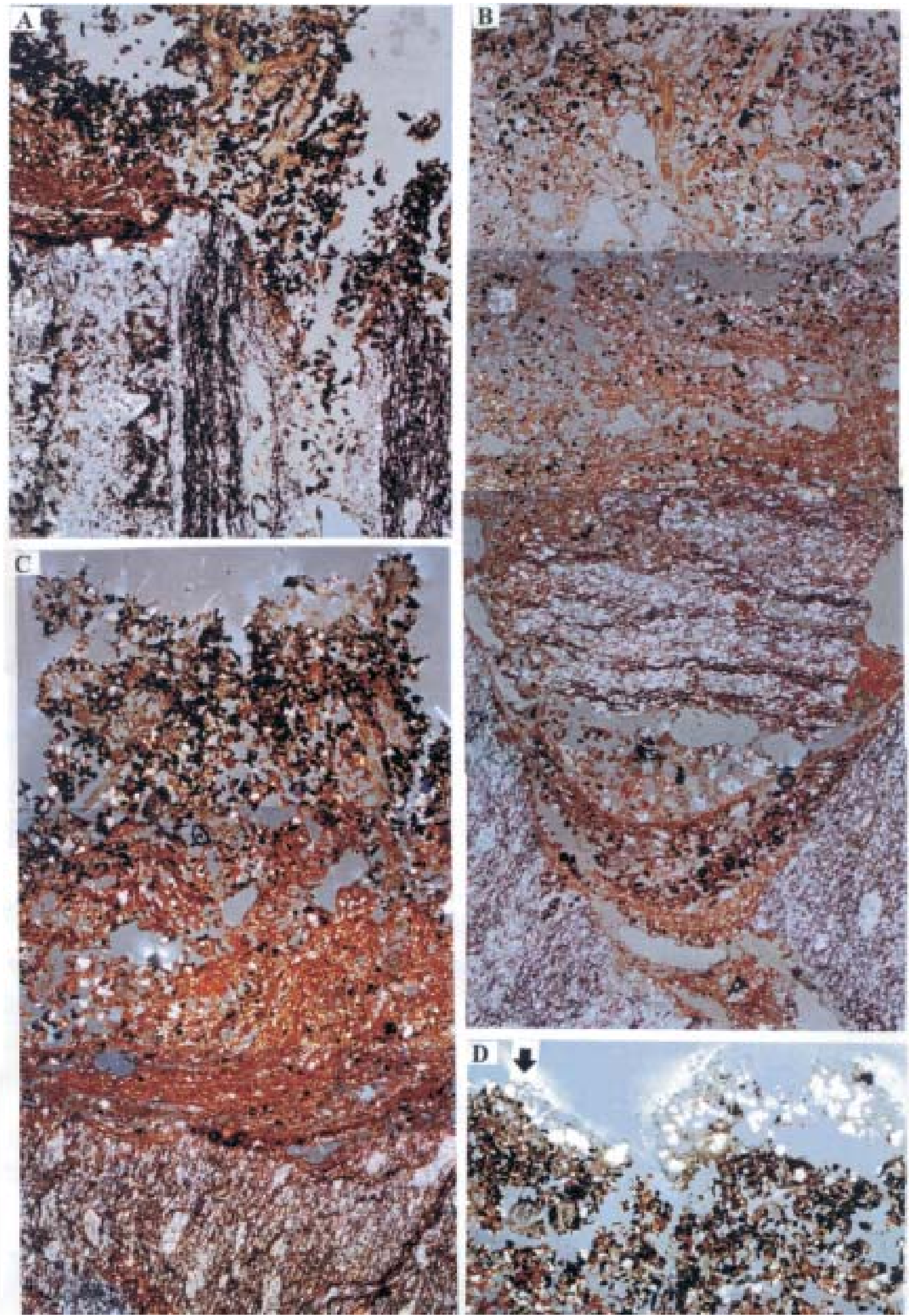

Figura 3 - Fotomicrografias das crostas biológicas sobre saprolitos de XT. A) banda biotítica/muscovítica (à direita), onde a penetração por materiais bioturbados é mais profunda, em relação à banda quartzosa, à esquerda.; B) bolsões fraturados com recobrimento de sucessivas deposições organo-ferruginosas (sápricas), fragmentos de saprolito e nova sucessão micropedogenética, até a crosta biológica superficial, rica em briófitas (topo); C) feições micropedogenéticas de forte redistribuição de complexos descontinuamente sobreposta ao saprolito; D) tapetes de líquens e algas (algalmatts) descontínuos sobre horizonte sáprico mais evoluido. 
No itabirito (IT), observa-se uma cobertura biológica mais rala e descontínua (Figura 2 D), com áreas estruturadas onde há uma abundância de micropartículas oxídicas, provavelmente de hematita e magnetita opacas, sobrepostas à matriz fortemente ferruginizada e cimentada, abaixo (seta).

Em Morro Velho (MV), o micro-horizonte fíbrico (Fox, 1985) é mais delgado e descontínuo em relação ao gnaisse (Figura $2 \mathrm{~B}$ ), sendo observada a presença de uma sub-camada mais estruturada com características sápricas e ausência de restos vegetais reconhecíveis. Nas partes mais ferruginizadas da crosta em MV, observa-se uma concentração de areia fina quartzosa e uma delgada cobertura de algalmatts (líquens e algas) descontínua, com certa fluorescência em luz polarizada (Figura 2E). Abaixo, verifica-se uma zona maciça e pouco estruturada (Figura $2 \mathrm{G}$ ), sendo a crosta biológica de penetração muito limitada, em relação às crostas instaladas sobre os demais saprolitos estudados.

A maior diversidade de feições micropedogenéticas foi observada no xisto (XT), concordando com os maiores teores de carbono. Algumas das feições de destaque são mostradas na Figura 3. Seqüências de crostas biológicas com profundidades de até $14 \mathrm{~mm}$ foram observadas em microscópio.

A alternância de microbandas (fácies) mais quartzosas com outras micáceas mostrou-se claramente um fator importante na ocorrência e tipologia das crostas. Na Figura 3 A, pode-se observar uma banda biotítica/muscovítica (à direita), onde a penetração por materiais bioturbados é mais profunda, em relação à banda quartzosa, à esquerda. Na parte micácea, há ocorrência de briófitas alongadas e pelotas fecais de microartropodos, enquanto na parte quartzosa um microambiente abaixo da crosta favoreceu a acumulação e precipitação de $\mathrm{Fe}$ (zona ferruginizada compacta, mais escura), como um verdadeiro "micro-ortstein", apresentando poros planares.

Essas feições micropedo-genéticas de forte redistribuição de complexos de Fe e Fe-MO na subcrosta mostram profundidades variáveis, de até $500 \mathrm{~mm}$, e estão sempre localizados sobre o saprolito, descontinuamente (Figura 3 C).

A ação microbiótica mostrou ainda feições de intensa micropedogênese, em bolsões fraturados (Figura 3 B), onde há um recobrimento de sucessivas deposições organo-ferruginosas (sápricas), fragmentos de saprolito e nova sucessão micropedogenética, até a crosta biológica superficial, rica em briófitas (Figura $3 \mathrm{~B}$, topo). Revestimentos ferruginosos são comuns também nas fraturas e planos de clivagem do saprolito micáceo (Figura 3 B e C, base). Tapetes de líquens e algas (algalmatts) descontínuos, coroam as partes mais microestruturadas, onde já é discernível o horizonte sáprico, mais evoluído (Figura $3 \mathrm{D}$ ).

\section{CONSIDERAÇÕES FINAIS}

Os resultados obtidos no presente trabalho revelam um efeito significativo da ciclagem biogeoquímica associada à ocorrência de crostas biológicas na disponibili-zação de elementos essenciais à instalação de plantas superiores na superfície exposta de saprolitos. A relevância deste fenômeno mostrou-se dependente das características físico-químicas do material parental, embora no caso de $\mathrm{K}$ e Fe a concentração da fração disponível na crosta ou na camada micropedogenizada subjacente, em todos os pontos, demonstre uma intensa mobilização destes elementos por influência biogeoquímica, sobrepujando a influência da natureza química do saprolito, normalmente acentuada em profundidade.

Independentemente de eventuais desbalanços químicos de macro e micronutrientes, toxidez ou presença de excesso de sais, as crostas biológicas recobriram de forma conspícua os saprolitos, sendo responsáveis por feições micropedo-genéticas evidentes e pelo processo de estruturação biológica da superfície exposta dos taludes. Este processo é capaz de alterar a dinâmica hídrica e erosiva da vertente, o que tem importante implicação geomorfológica e ambiental.

\section{REFERÊNCIAS BIBLIOGRÁFICAS}

BARKER, W.W. et al. (1998) Biogeochemical weathering of silicate minerals. In: Geomicrobiology, 391-428.

BROWN, D.H. (1976) Mineral uptake by lichens. In: D.H Brown, D.L. Hawksworth e R.H. Bailey (eds.) Lichenology: progress and problems 8. Proc. Int. Symp. University Bristol London, Academic Press Inc., 419-439.

CHENU, C. (1989) Influence of a fungal polysaccharide, scleroglucan, on clay microstructures. Soil Biol. Biochem., 21: 299-305.

CHENU, C. (1993) Clay - or sand - polysaccharide associations as models for the interface between micro-organisms and soil: water related properties and microstructure. Geoderma, 56: $143-156$.

DORIOZ, J.M. et al. (1993) The role of roots, fungi and bacteria on clay particle organization. An experimental approach. Geoderma, 56: 179-194.

EHRLICH, H.L. (1998) Geomicrobiology: its significance for geology. Earth-Science Reviews, 45: 45-60.

EMPRESA BRASILEIRA DE PESQUISA AGROPECUÁRIA (EMBRAPA) (1997) Manual de métodos de análise de solo.2ed. Rio de Janeiro, EMBRAPA/CNPS, 212 p.

EMERSON, W. W. (1995) The plastic limit of silte, surface soils in relation to their content of polysaccharide gel. Aust J. Soil Res., 33: 1-9.

FARIA, J. C. (1996) Dinâmica da água, comportamento térmico e selamento de um podzólico vermelho-amarelo, em relação ao controle de plantas invasoras. Viçosa, UFV, 75 p. (Tese M.Sc.)

FITZPATRICK, E. A (1993) Soil microscopy and micromorphology. Chichester, John Wiley, 304 p.

FOX, C. A. (1985) Micromorphological characterization of histosols. In: L. A. Douglas e M.L. Thompson (eds). Soil micromorphology and soil classification. Soil Science Society of America, Special Publication 15, Madson, 85-104 pp.

GOMES, M. A. F. (1988) Intemperismo de anfibolitos e sua contribuição à gênese de solos de duas províncias metamórficas do Estado de Minas Gerais. Viçosa, UFV, 138p. (Tese M.Sc.) 
ISKANDAR, I. K. e SYERS, J. K. (1972) Metal-complex formation by lichen compounds. Journal of Soil Science, 23: 255-265.

JONES, D. et al. (1987) Effects of lichens on mineral surfaces. In: D. R. Houghton e E. Smith (eds.) Biodeterioration 7. Proc. $7^{\text {th }}$ Int. Biodeterioration Symp., 1987. Amsterdam, Elsevier, 129-134.

LADEIRA, E. A. (1980) Gênese de ouro na mina de Morro Velho e no distrito de Nova Lima, Minas Gerais, Brasil. In: Congresso Brasileiro de Geologia, 31, Camboriú, 1980. Anais, Camboriú, SBG, 371p.

LYNCH, J. M. e ELLIOTT, L. F. (1983) Aggregate stabilization of vulcanic ash and soil during microbial degradation of straw. Applied and Environmental Microbiology, 45: 13981401 .

MARTIN, J. P. e WAKSMAN, S. A. (1940) Influence of microorganisms on soil aggregation and erosion. Soil Science, 50: 29-47.

NEUMAN, C. M. et al. (1996) Wind transport of sand surfaces crusted with photoautotrophic microorganisms. Catena, 27: 229-247.

PEELE, T. C. e BEALE, O. W. (1941) Effect on runoff and erosion of improved aggregation resulting from the simulation of microbial activity. Soil Science Society of America, 6: 176-182.
PÉREZ, F. L. (1997) Microbiotic crusts in the high equatorial Andes, and their influence on paramo soils. Catena, 31: $173-198$.

TISDALL, J. M. e OADES, J. M. (1982) Organic matter and water-stable aggregates in soils. Journal of Soil Science, 33: 141-163.

VILES, H. e PENTECOST, A. (1994) Problems in assessing the weathering action of lichens with an example of epiliths on sandstone. In: D.A. Robinson e R.B.G. Williams (eds.) Rock weathering and landform evolution. Chichester, John Wiley \& Sons Ltd, 99-116.

WEED, S. B. et al. (1969) Weathering of mica by fungi. Soil Science Society of America, 33: 702-706.

WIERZCHOS, J. e ASCASO, C.(1998) Mineralogical transformation of bioweathered granitic biotite, studied by HRTEM: evidence for a new pathway in lichen activity. Clays and Clay Minerals, 46: 446 - 452.

WILLIAMS, R.B.G. e ROBINSON, D.A. (1994) Weathering flutes on siliceous rocks in Britain and Europe. In: D.A. Robinson e R.B.G. Williams (eds.) Rock weathering and landform evolution. Chichester, John Wiley \& Sons Ltd, 413-432.

ZIMMERMAN, W. J. (1993) Microbial biotechnology and applications in agriculture. In: F. Blaine Metting, Jr.(ed) Soil microbial ecology. Aplications in agricultural and environmental management. New York, Marcel Dekker, Inc.,457-479. 